\title{
Photocatalytic transformation in aerogel-based optofluidic microreactors
}

\section{Yaprak Özbakır, Alexandr Jonáš, Alper Kiraz, Can Erkey}

Yaprak Özbakır, Alexandr Jonáš, Alper Kiraz, Can Erkey, "Photocatalytic transformation in aerogel-based optofluidic microreactors," Proc. SPIE 10535, Integrated Optics: Devices, Materials, and Technologies XXII, 105352C (23 February 2018); doi: 10.1117/12.2292309

SPIE. Event: SPIE OPTO, 2018, San Francisco, California, United States 


\title{
Photocatalytic transformation in aerogel-based optofluidic microreactors
}

\author{
Yaprak Özbakır ${ }^{\mathrm{a}}$, Alexandr Jonáś ${ }^{\mathrm{b}}$, Alper Kiraz ${ }^{\mathrm{c}, \mathrm{d}}$ and Can Erkey ${ }^{\mathrm{a}}$ \\ ${ }^{a}$ Department of Chemical and Biological Engineering, Koc University, 34450 Sarıer, Istanbul, \\ Turkey \\ ${ }^{\mathrm{b}}$ Department of Physics, Koc University, 34450 Sariyer, Istanbul, Turkey \\ ${ }^{\mathrm{c}}$ Institute of Scientific Instruments of the ASCR, v.v.i., Academy of Sciences of the Czech Republic, \\ Královopolská 147, 61264 Brno, Czech Republic \\ ${ }^{\mathrm{d}}$ Department of Electrical and Electronics Engineering, Koc University, 34450 Saryer, Istanbul, \\ Turkey
}

\begin{abstract}
Here, we demonstrate a new type of microphotoreactor formed by a liquid-core optofluidic waveguide fabricated inside aerogel monoliths. It consists of microchannels in a monolithic aerogel block with embedded anatase titania photocatalysts. In this reactor system, aerogel confines core liquid within internal channels and, simultaneously, behave as waveguide cladding due to its extremely low refractive index of $\sim 1$. Light is confined in the channels and is guided by total internal reflection (TIR) from the channel walls. We first fabricated L-shaped channels within silica aerogel monoliths $\left(\rho=0.22 \mathrm{~g} / \mathrm{cm}^{3}, \mathrm{n}=1.06\right)$ without photocatalyst for photolysis reactions. Using the light delivered by waveguiding, photolysis reactions of methylene blue (MB) were carried out in these channels. We demonstrated that MB can be efficiently degraded in our optofluidic photoreactor, with the rate of dye photoconversion increasing linearly with increasing power of incident light. For photocatalytic transformation in this reactor system, titania particles were successfully embedded into the mesoporous network of silica aerogels with varying amount of the titania in the structure from $1.7 \mathrm{wt} \%$ to $50 \% \mathrm{wt}$. The presence of titania and its desired crystalline structure in aerogel matrix was confirmed by XRF, XRD patterns and SEM images. Band gap of silica-titania composites was estimated from Tauc plot calculated by Kubelka-Munk function from diffuse reflectance spectra of samples as near expected value of $\approx 3.2 \mathrm{eV}$. Photocatalytic activity and kinetic properties for photocatalytic degradation of phenol in the channels were investigated by a constant flow rate, and longer-term stability of titania was evaluated.
\end{abstract}

Keywords: aerogels, porous nanomaterials, optofluidic waveguides, microchannels, total internal reflection, photocatalyst, photochemistry.

\section{INTRODUCTION}

Optofluidic microreactors where photochemical reactions are carried out in solutions in microfluidic channels in the presence of light are attracting increasing attention for a wide variety of applications ranging from detection of toxic chemicals to diagnosis of biological entities [1-4]. The maximized overlap between light and samples in small volumes enables highly-efficient photochemistry at optical powers less than in conventional systems with improved spatial irradiation homogeneity and better light penetration through the reaction volume compared to conventional large-scale reactors [3, 5-7].

Various types of optofluidic microreactors have been described in the literature. A variety of optofluidic microreactors reactors have been described in the literature which consist of microchannels in a flat plate, which is covered by a 
window. Light is delivered to such microreactors by external irradiation through planar transparent glass covers. Li et al. [8] designed a micro-capillary based microreactor by using capillaries with an inner diameter of $200 \mu \mathrm{m}$. The inner wall of the capillary was coated with a $\mathrm{TiO}_{2}$ (titania) $/ \mathrm{SiO}_{2}$ film. The degradation of aqueous methylene blue in the capillary was investigated by externally irradiating the capillary with a light source from the outside. However, this external irradiation may not be ideal for utilization of the light since a significant fraction of light was absorbed by the outer part of the photocatalyst layer, which reduced the efficiency of the photocatalytic degradation. In some other studies, microchannels were fabricated on glass, polymer and ceramic substrates using several etching methods such as photolithography, dry/wet etching and micro/nano imprinting. For instance, Matsushita et al.[9] designed a single straight microchannel the bottom of which was covered with a $\mathrm{TiO}_{2}$ thin film loaded with Pt particles to carry out photodegradation, reduction of organic compounds as well as amine $N$-alkylation by externally irradiating the reaction system. Gorges et al.[10] used a microreactor with 19 branched parallel microchannels. A nanoporous $\mathrm{TiO}_{2}$ film was immobilized and a UV-LED array was fixed above the area of the branched channels. Furthermore, planar microreactors have been utilized for further improvement of the light utilization of the surface of microfluidic chips. Lei et al.[11] designed a planar microreactor by using two nanoporous $\mathrm{TiO}_{2}$-coated glasses as the cover and substrate and a $100 \mu \mathrm{m}$ thick epoxy layer as the spacer and sealant. Similarly, Ahsan et al.[12] utilized a planar microfluidic platform to study the kinetics of photocatalytic water-splitting. They used glass slides, which were coated with a $\mathrm{Pt}-\mathrm{TiO}_{2}$ photocatalyst prepared by a sol-gel method. Furthermore, Meng et al. [13] developed a new approach for fabrication of the planar microreactor by using electrospun nanofibrous $\mathrm{TiO}_{2}$ sealed between polydimethylsiloxane (PDMS) substrate and a glass slide.

An alternative way to deliver light to an optofluidic microreactor through the reaction medium may be using optofluidic waveguides. In this approach, the external wall of the microchannel should be made out of a suitable material which confines the reaction medium and also behaves like waveguide cladding. Aerogels are highly porous nanostructured materials that can be produced as macroscopic monoliths with unique properties such as extremely low refractive index, high surface area to volume ratio, and high porosity $[14,15]$. These properties, particularly the low refractive index of $\sim 1.05$, make aerogels a remarkable solid-cladding material without any additional coating since almost all liquids have refractive indices exceeding that of aerogel. Aerogel-based optofluidic waveguides are constructed by opening microchannels inside monolithic aerogel blocks. Following surface treatment, the channels are filled with a suitable liquid that serves as the core liquid without any restriction on the type of liquid and the type of aerogel that can be used, as long as the liquid can be confined inside the aerogel block without penetrating its network. Due to the low refractive index of aerogels, light can be uniformly propagated by total internal reflection (TIR) in the reaction medium inside the channel in an aerogel with low losses similar to conventional optical fibers $[14,16]$.

Photocatalytic reactions are another interesting area for the potential application of aerogel-based optofluidic waveguides. Their high porosity and high surface area with interconnected open pore structure, combined with a fully tunable three-dimensional structure, make aerogels effective for photocatalyst deposition in their structure, thus potentially improving photocatalytic activity. Microchannel walls in an aerogel monolith are porous and photocatalysts can be immobilized within these pores with good adhesion to the solid network. The light can be partially absorbed in the near-surface region of the photocatalysts immobilized in the pores of the wall. Thus, the reactants can possibly be converted into desired products on the catalyst surface, effectively driving the reactions.

Based on this concept, we demonstrate a new type of aerogel-based optofluidic microreactor in this study. It consists of a single channel in a monolithic aerogel block with embedded photocatalysts for carrying out photochemical reactions using the light delivered to the reaction medium by an optical waveguide based on TIR. In this reactor system, aerogel confines the liquid reactant within internal channels and, simultaneously, behaves as waveguide cladding due to its extremely low refractive index of $\sim 1$. Light is confined in the channels and is guided in a controlled manner by the aid of liquid core optical waveguides based on TIR from the channel walls. We successfully show that our photoreactor is well suited for light-driven photolysis of a model organic compound - methylene blue (MB) dye - by the light guided along the full length of the channel and we characterize the efficiency of dye photoconversion as a function of the incident light intensity. We also reveal that our photomicroreactor is well suited for photocatalytic degradation of phenol over titania. Photocatalytic activity and kinetic properties for photocatalytic degradation of a compound (phenol) in the fabricated channels using light delivered by waveguiding are investigated by a constant flow rate, and longer-term stability of titania is evaluated. 


\section{MATERIALS AND METHODS}

\subsection{Synthesis of silica aerogels}

Silica aerogels were synthesized using a two-step sol-gel process followed by aging of so-called alcogels in a Tetraethylorthosilicate (TEOS) (98\%; Sigma Aldrich) containing aging solution and extraction of the solvent from the porous alcogel network, or drying of the alcogel. TEOS was used as the silica precursor and it was initially hydrolyzed with water in the presence of ethanol ( $\geq 98 \%$; Sigma Aldrich) as the cosolvent and $\mathrm{HCl}(0.06 \mathrm{M}$ in ethanol; Riedel-de Haen) as the acid catalyst. After addition of the acid catalyst, the solution was continuously stirred at room temperature for $1 \mathrm{~h}$ and transformed to a sol. Subsequently, $\mathrm{NH}_{4} \mathrm{OH}(0.1 \mathrm{M}$ in ethanol; Sigma Aldrich) was added to the sol as the base catalyst to adjust the solution $\mathrm{pH}$ such that the rate of silanol condensation increased eventually leading to gelation of the solution. The molar ratio of TEOS/ethanol/water/HCI $/ \mathrm{NH}_{4} \mathrm{OH}$ used in the alcogel synthesis was 1:4:3:0.0023:0.009. After gelation, the resulting alcogels were subsequently soaked in aging solution (40 v/v \% TEOS, $10 \mathrm{v} / \mathrm{v} \%$ water, $50 \mathrm{v} / \mathrm{v} \%$ ethanol) at $50^{\circ} \mathrm{C}$ in an oven for $24 \mathrm{~h}$. The samples were further kept in TEOS aging solution at room temperature for 3 days. The alcogels were washed in fresh ethanol for 3 days to remove any impurities and water remaining in the pores of the alcogels. As the last step, the alcogels were dried at $40{ }^{\circ} \mathrm{C}$ and 100 bar with supercritical $\mathrm{CO}_{2}\left(\mathrm{scCO}_{2}\right)$ for $6 \mathrm{~h}$. The monolithic and crack-free aerogels were produced from the same batch.

\subsection{Synthesis of silica-titania aerogels}

Silica-titania aerogels were synthesized by addition of anatase titania powder into the sol during the sol-gel step, and followed by gelation and extraction of the solvent from the porous alcogel network. The sol was prepared as described in Section 2.2 with the molar ratio of 1:4:3:0.0023:0.009 (TEOS/ethanol/water/HCI/ $\left.\mathrm{NH}_{4} \mathrm{OH}\right)$ and the titania particles was added to obtain the weight percent of titania in silica in a range from $1.7 \mathrm{wt} \%$ to $50 \mathrm{wt} \%$. The solution was continuously stirred at room temperature for $2 \mathrm{~h}$ and transformed to a sol comprising uniformly dispersed titania particles. Afterwards, $\mathrm{NH}_{4} \mathrm{OH}(0.25 \mathrm{M}$ in ethanol; Sigma Aldrich) was added to the sol as the base catalyst to initiate condensation reactions and eventually gelation of the solution occurred. The resulting gels were immersed in 50:50 (v\%) solution of ethanol and water at $50{ }^{\circ} \mathrm{C}$ in the oven for $24 \mathrm{~h}$. Following the aging step, the alcogels were washed in fresh ethanol for 3 days to exchange solvent remaining in the pores to ethanol. Lastly, the silica-titania alcogels were dried at $40{ }^{\circ} \mathrm{C}$ and 100 bar with supercritical $\mathrm{CO}_{2}\left(\mathrm{scCO}_{2}\right)$ for $6 \mathrm{~h}$.

\subsection{Surface modification with HMDS}

The obtained aerogels are hydrophilic due to the presence of the polar hydroxyl $(\mathrm{OH})$ groups within their porous framework which promote high capillary stress and water adsorption once they are in contact with water. Therefore, the aerogel samples were transformed into the hydrophobic ones by replacing the hydrophilic surface groups with hydrophobic groups. The surface of the synthesized silica aerogel monoliths was rendered hydrophobic using hexamethyldisilizane (HMDS) ( $\geq 98 \%$; Merck) vapor at $110^{\circ} \mathrm{C}$ as the surface modification agent in a tightly sealed container. Monolithic, crack-free hydrophobic aerogels were obtained. After silanization of surface silanol groups with HMDS vapor, hydrophobicity of the samples was determined by measuring water contact angle on the silanized aerogel surface.

\subsection{Characterization of silica aerogels}

Average pore size, pore size distribution and BET surface area of the samples were determined using nitrogen physisorption (Micromeritics ASAP 2020). Samples were initially left to degas at $300{ }^{\circ} \mathrm{C}$ under vacuum for 1 day to remove remaining impurities from the surface. The pore analysis was performed using $\mathrm{N}_{2}$ adsorption/desorption isotherms with a relative pressure $\left(\mathrm{P} / \mathrm{P}_{0}\right)$ ranging from $10^{-7}$ to $1 \mathrm{~atm}$. The surface area of the samples was determined by Brunauer-Emmett-Teller (BET) method and the pore volume and pore size distributions were determined by Barrett-Joyner-Halenda (BJH) analysis from the $\mathrm{N}_{2}$ adsorption-desorption isotherms. Total pore volume of the sample was determined by converting adsorbed $\mathrm{N}_{2}$ volume at STP to liquid $\mathrm{N}_{2}$ volume at $77 \mathrm{~K}$. Bulk density of the monolithic 
sample was determined by dividing its mass by its final volume, which was obtained by measuring the physical dimensions of the aerogel using a caliper. Refractive index of the aerogel was measured by characterizing laser beam refraction from the aerogel block placed on a goniometer stage as demonstrated in our recent study [16].

\subsection{Experimental setup for photochemical reactions}

Figure 1 shows a schematic diagram of the experimental setup that was employed for studying model photochemical reactions - degradation of $\mathrm{MB}$ in aqueous solutions and phenol in aqueous solutions contained inside the fabricated microchannel in the aerogels. The hydrophobic silica aerogel block with the inclined L-shaped channel was mounted on an adjustable metal holder and it was connected to a Union Tee adapter. Central port of the Union Tee was then connected to a syringe and the remaining end of the Union Tee was used to insert a solarization-resistant multimode optical fiber. This fiber served for delivering light to the reaction volume and its end was held at a fixed position at the channel entrance. The channel was filled with the aqueous MB solution using the syringe. In order to initiate light-driven degradation of MB, we used a laser beam from a femtosecond-pulsed, tunable laser light source (Coherent Chameleon) with a maximal output power of $4 \mathrm{~W}$. The laser beam at $388 \mathrm{~nm}$ was coupled into the free end of the multimode optical fiber with the aid of an objective lens. The input power coupled into the optofluidic waveguide could be adjusted from the laser source. The intensity of light transmitted through the waveguide was visually monitored and measured at the outlet of the channel by a laser power meter. In order to monitor the progress of photodegradation of $\mathrm{MB}$, samples with a volume of $10 \mu \mathrm{L}$ were collected at certain time intervals from the end of the channel by a micropipette and subsequently analyzed by Nanodrop ND-1000 Spectrophotometer for absorbance-based quantification of MB concentration. Similarly, silica-titania composite blocks with a cylindrical channel inside were placed connected to the Union Tee for photocatalytic degradation of phenol over titania embedded in the channel walls. The channel was filled with a phenol solution with a concentration of $\sim 680 \mu \mathrm{M}$ by a syringe pump at a constant flow rate. The operating wavelength of the laser was tuned to $366 \mathrm{~nm}$ and the light was coupled by the optical fiber from the same opening where the solution was filled. The exit stream of the channel was collected at certain time intervals as a function time. The concentration of the collected samples was analyzed as described above. Light-guiding experiments with aerogel-based optofluidic waveguides were also performed using the same experimental set-up. The channel fabricated within the aerogel monolith was first filled by water and illuminated by laser light at $488 \mathrm{~nm}$ coupled into the horizontal channel through the optical fiber. The light power at channel input and output was measured.

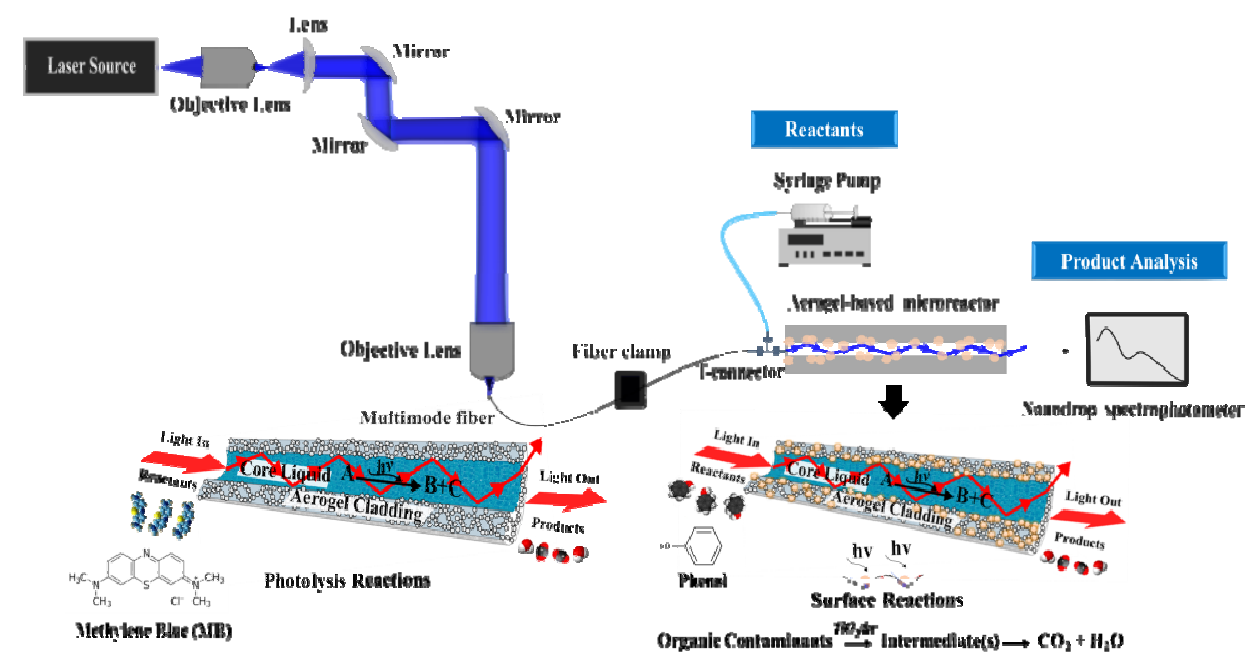

Figure.1. Schematics of experimental set-up used in characterization of light guiding and photochemical reactions in aerogel-based optofluidic microreactors. 


\section{RESULTS}

\subsection{Properties of synthesized aerogel samples}

Synthesis of aerogel monoliths with high mechanical strength and sufficiently low refractive index to serve as a solid cladding material for optofluidic waveguides is a critical step for fabricating our photoreactors [14, 16]. The aerogels need to be sufficiently strong to withstand manual drilling of relatively long channels without compromising their shape and nanoporous structure. This was accomplished by using an aging process of the alcogels in a solution containing the silica precursor. After aging, the resulting sample had a density of $0.22 \mathrm{~g} / \mathrm{cm}^{3}$. The critical angle of the laser beam refracted from the aerogel - air interface was measured to be $71^{\circ}$, which translated into the refractive index of $1.060 \pm 0.007$ at $632.8 \mathrm{~nm}$, much lower than the refractive index of an aqueous solution. Long channels $(\sim 7.5 \mathrm{~cm})$ as straight and inclined L-shaped channels $(2.1 \mathrm{~mm} \mathrm{X} 4.2 \mathrm{~cm})$ could be fabricated by drilling. Water-filled optofluidic waveguides in synthesized aerogels yielded high numerical aperture values $(\sim 0.8)$. Efficient guiding of light by total internal reflection in the aqueous MB solution-filled channel in aerogels was visually revealed and characterized by monitoring power at channel output (Figure 2). The characterization of transmission indicated that propagation losses were lower than $2.5 \mathrm{~dB} \mathrm{~cm}^{-}$, demonstrating that liquid-core waveguides with drilled aerogel represent an attractive alternative in applications targeting controlled light routing along arbitrary paths.
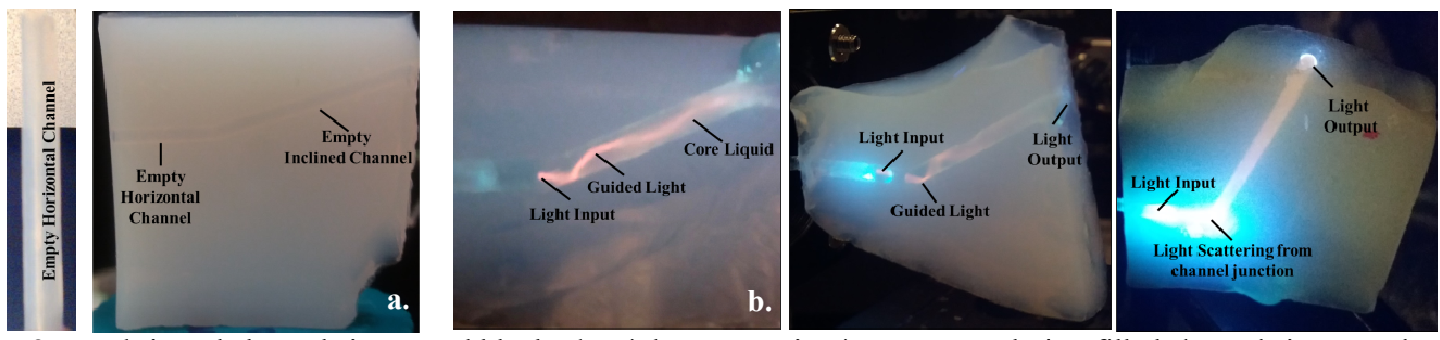

Figure 2. a. Fabricated channels in aerogel blocks. b. Light propagation in aqueous solution-filled channels in aerogel blocks.

Titania particles were successfully introduced into the mesoporous network of silica aerogels by addition during sol-gel step of the aerogel synthesis providing their crack-free and monolithic structures. The presence of titania and its desired crystalline structure in aerogel matrix was confirmed by scanning electron microscope (SEM) images and XRD patterns. Figure 3 represents SEM images of the composites samples with $6.5 \mathrm{wt} \% \mathrm{TiO}_{2}$ and $50 \mathrm{wt} \% \mathrm{TiO}_{2}$, respectively with scale bars of $200 \mathrm{~nm}$ and $1 \mu \mathrm{m}$. The images revealed that anatase titania particles formed agglomerated titania clusters (which appear as white spheres) during sol-gel step and these nanometer titania clusters have been homogenously dispersed on the silica network (which appears as gray areas).
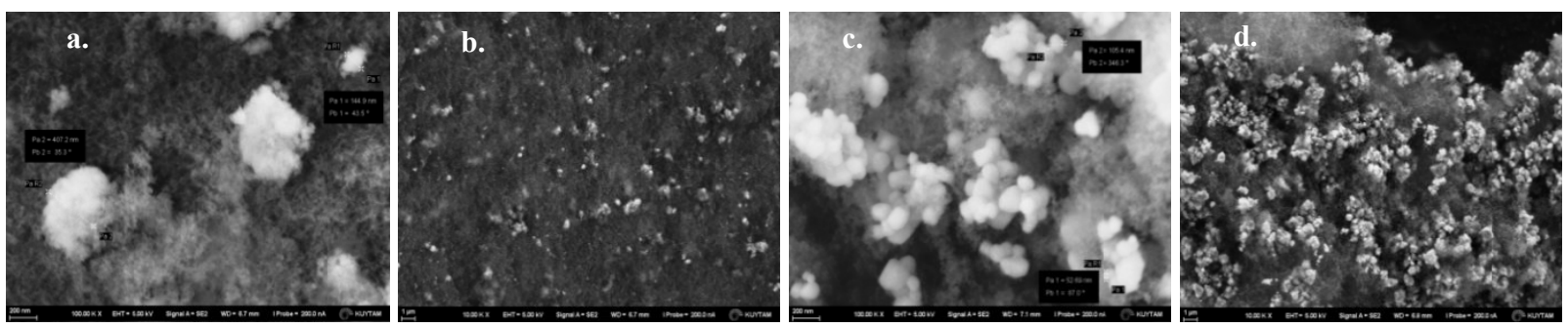

Figure 3. a. SEM images of silica-titania aerogel (6.5 wt $\left.\% \mathrm{TiO}_{2}\right)$, scale bar, $200 \mathrm{~nm}$. b. SEM images of silica-titania aerogel $\left(6.5 \mathrm{wt} \% \mathrm{TiO}_{2}\right)$, scale bar, $1 \mu \mathrm{m}$. c. SEM images of silica-titania aerogel $\left(50 \mathrm{wt} \% \mathrm{TiO}_{2}\right)$, scale bar, $200 \mathrm{~nm}$. d. SEM images of silica-titania aerogel $\left(50 \mathrm{wt} \% \mathrm{TiO}_{2}\right)$, scale bar, $1 \mu \mathrm{m}$. 
The synthesized silica-titania aerogels attained their high porosity and pore volume as well as high surface area. Table 1 shows the pore volume and BET surface area of the composites and a typical native silica aerogel $\left(\rho \approx 0.15 \mathrm{~g} / \mathrm{cm}^{3}\right)$. The surface area of the samples decreases with increase of the titania amount in the network. The surface area decreased from $949 \mathrm{~m}^{2} / \mathrm{g}$ for the native silica aerogel to $443 \mathrm{~m}^{2} / \mathrm{g}$ for the sample with $50 \mathrm{wt} \% \mathrm{TiO}_{2}$. Similarly, the pore volume of the native silica aerogel decreased from $4.3 \mathrm{~cm}^{3} / \mathrm{g}$ to $2.9 \mathrm{~cm}^{3} / \mathrm{g}$ with $50 \mathrm{wt} \% \mathrm{TiO}_{2}$. However, Figure $4 \mathrm{a}$ indicates that the pore diameter of the samples does not exhibit significant changes as compared to pore diameter of the native silica aerogel. The $\mathrm{N}_{2}$ adsorption/desorption isotherms of the silica aerogel and samples are additionally given in Figure 4b. It is clearly observed that both the silica aerogel and samples show type IV isotherms with distinct capillary condensation steps, which shows that they are typical mesoporous materials with three-dimensional network.

Table 1. Pore Characteristics of the Silica-Titania Composites

\begin{tabular}{ccc}
\hline $\begin{array}{c}\text { Sample } \\
(w t \%)\end{array}$ & $\begin{array}{c}S_{B E T} \\
\left(\mathrm{~m}^{2} / g\right)\end{array}$ & $\begin{array}{c}V_{\text {pore }} \\
\left(\mathrm{cm}^{3} / \mathrm{g}\right)\end{array}$ \\
\hline 0 & 949 & 4.3 \\
1.7 & 890 & 4.0 \\
7.5 & 948 & 4.3 \\
46 & 494 & 3.2 \\
50 & 443 & 2.9 \\
\hline
\end{tabular}

Furthermore, band gap of the silica-titania composites was estimated from Tauc plot in Figure 4c calculated by KubelkaMunk function, $F(R)$, in Eq. (1) from diffuse reflectance (R) spectra of samples using UV-visible reflectance spectroscopy. Using this technique, the anatase titania band gap was observed near expected value of $\approx 3.2 \mathrm{eV} \mathrm{[17].}$

$$
f(h v)=\frac{(1-R(h v))^{2}}{2 R(h v)}
$$
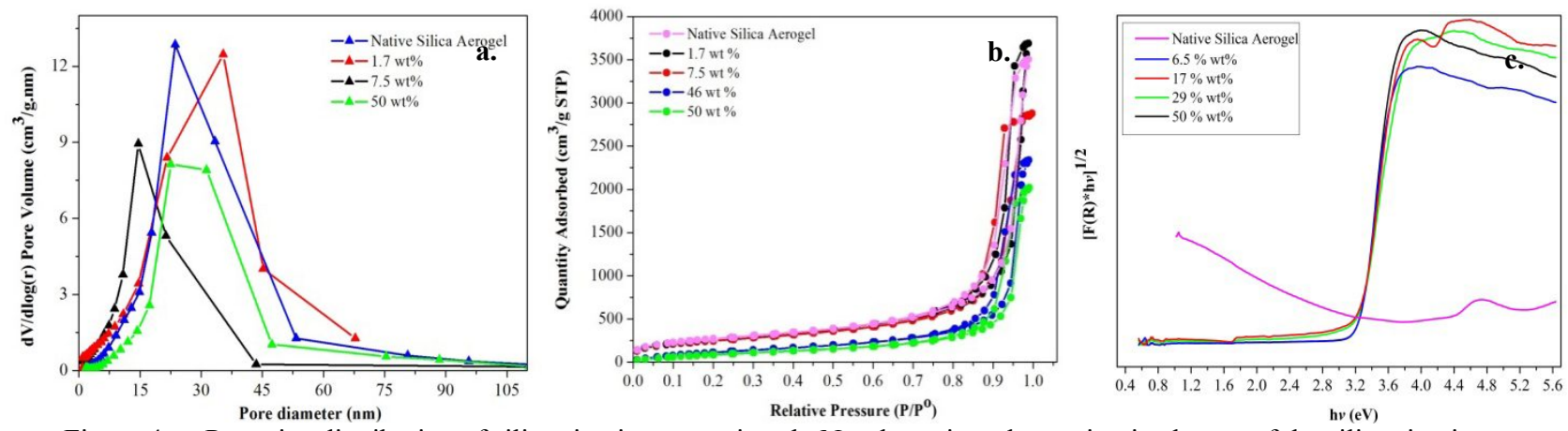

Figure 4. a. Pore size distribution of silica-titania composites. b. $\mathrm{N}_{2}$ adsorption-desorption isotherms of the silica-titania composites. b. c. Tauc plot of $\left[\mathrm{F}(\mathrm{R})^{*} h v\right]^{1 / 2}$ versus photon energy, $h v$, to estimate bandgap and absorption edge of the silicatitania composites.

Figure 5 represents the XRD patterns of the silica-titania composite aerogels with varying amount of $\mathrm{TiO}_{2}$. The silica aerogel is amorphous without any diffraction peaks, whereas the silica-titania composite aerogels show broad diffraction peaks at $25^{\circ}, 38^{\circ}, 48^{\circ}, 54^{\circ}, 55^{\circ}, 63^{\circ}, 69^{\circ}, 70^{\circ}$, and $75^{\circ}$, which correspond to (101), (004), (200), (105), (211), (204), (116), (220), and (215) reflection planes of anatase titania, respectively [18]. Figure 5 reveals that the peaks corresponding to the anatase phase become more intense with increase of the amount of titania. 

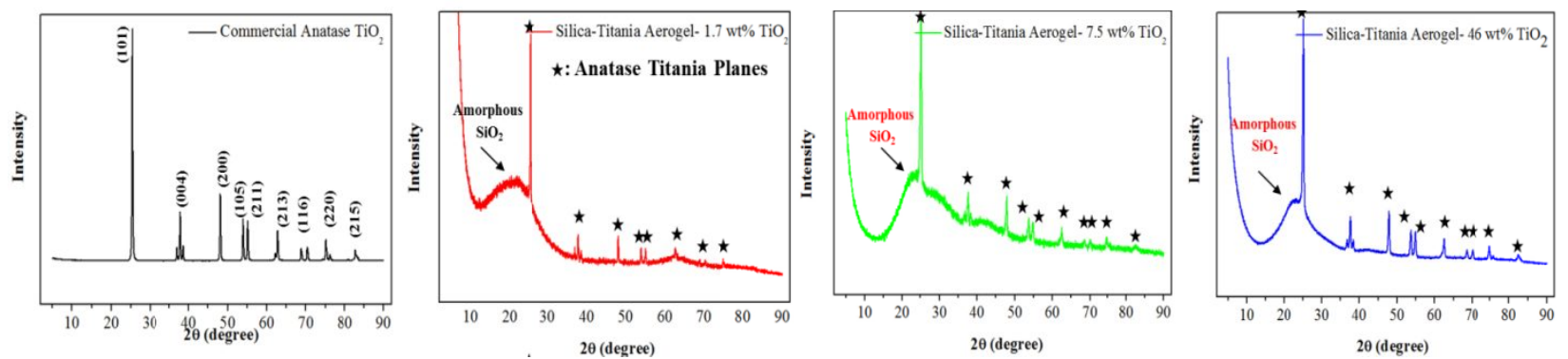

Figure 5. XRD patterns for silica aerogel and silica-titania composite aerogels with varying $\mathrm{TiO}_{2}$ amount.

\subsection{Photodegradation of organic compounds in aerogel-based microreactors}

Methylene Blue (MB) was chosen as a model organic compound to evaluate the performance of our aerogel-based photoreactors with integrated optofluidic waveguide in photochemical reactions. An aqueous solution of MB with a concentration of $36 \mu \mathrm{M}$ was loaded into an L-shaped silica aerogel channel. An L-shaped microchannel was chosen instead of a straight microchannel in order to make sure that the sample of the MB solution taken from the end of the reactor was exposed only to the light guided by TIR and not to the light emitted directly from the input optical fiber. Accordingly, light-induced degradation of MB occurred only due to waveguiding in the microchannel. Since MB might gradually adsorb on the surface of the aerogel, the concentration of MB solution collected from the channel at various times after starting the experiment might be lower than the initial concentration of the solution due to adsorption rather than due to photolytic degradation. In order to find out if there was a decrease in MB concentration due to adsorption, aerogel channel filled with MB solution was first kept in the dark for a certain period of time. The samples were periodically collected from the end of the channel and the concentration of MB in these samples was measured using Nanodrop Spectrophotometer. Figure 6a shows that the concentration of MB solution in the dark remained almost constant for 120 min with a very little variation, thus indicating that MB was not adsorbed on the channel surface. Subsequently, light was coupled into the aerogel channel filled with MB solution with initial concentration of $36 \mu \mathrm{M}$ and propagated to the end of the channel by TIR-assisted waveguiding. Under these experimental conditions, MB could be degraded along the full length of the channel by the guided light. The degree of photodegradation then depended on the light exposure time and the incident light power. Figure $6 \mathrm{~b}$ demonstrates that the concentration of MB samples collected from the channel end decreased with time under illumination with a constant power of the incident light. In particular, within 60 minutes, the concentration of $\mathrm{MB}$ decreased to $\sim 6 \mu \mathrm{M}$ under the exposure with the highest incident light power of $82 \mathrm{~mW}$ while it reduced to $\sim 16 \mu \mathrm{M}$ with the incident light power of $41.2 \mathrm{~mW}$ and to $\sim 19 \mu \mathrm{M}$ under the exposure with the incident light power of $21.2 \mathrm{~mW}$. The relative conversion of the MB dye compound was calculated from Eq. 2:

$$
\text { Conversion }(\%)=\frac{C_{0}-C_{t}}{C_{o}} \times 100
$$

As shown in Figure $6 \mathrm{~b}$, the relative conversion of MB dye increased linearly with the increasing power of the incident light within the studied power range. Specifically, after a 60-minute exposure, the relative photolytic conversion of MB was obtained as $48 \%, 56 \%$ and $83 \%$ at the end of 60 minutes, in the order of increasing incident power. 

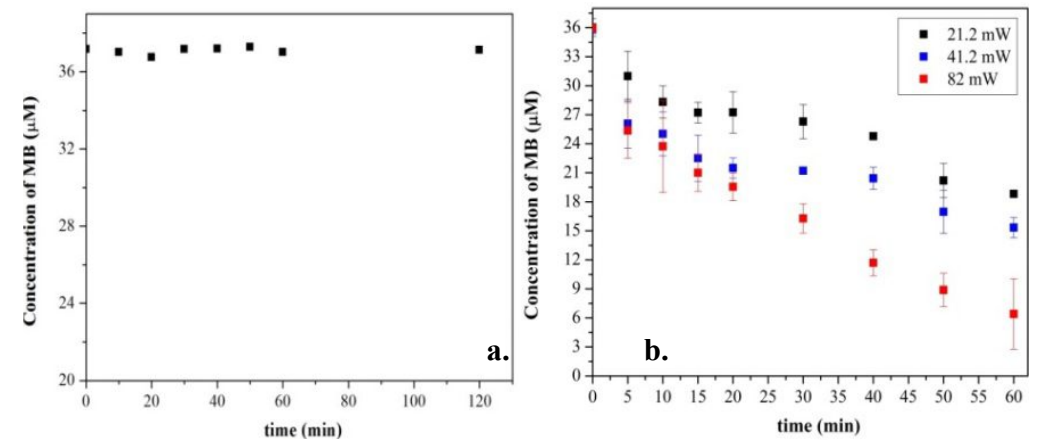

Figure 6. a.Time variation of the concentration of aqueous MB solution contained in the silica aerogel channel kept in the dark. The initial concentration of MB was $36 \mu \mathrm{M}$. b. Time variation of the concentration of aqueous MB solution contained in the silica aerogel channel under illumination at $388 \mathrm{~nm}$ with varying power of the incident light. The initial concentration of MB was 36 $\mu \mathrm{M}$. The incident power values were measured before coupling the light into the input optical fiber. (Channel size: $2.1 \mathrm{~mm}$ X 4.2 $\left.\mathrm{cm}, \mathrm{C}_{\mathrm{A}}{ }^{\mathrm{o}}=36 \mu \mathrm{M}\right)$

Photocatalytic activity and kinetic properties for photocatalytic degradation of phenol in aqueous solutions in the fabricated channels using light at $366 \mathrm{~nm}$ delivered by waveguiding were investigated by a constant flow rate, and longer-term stability of titania was evaluated. Concentration of the solution at the exit stream of the channel in dark was firstly investigated to explore if the phenol was adsorbed on the channel walls. Figure 7a shows that concentration of phenol solution remained almost constant in dark for $60 \mathrm{~min}$ with a little variation. The photodegradation of phenol was then investigated by photolysis experiments at $366 \mathrm{~nm}$ in a native silica aerogel monolith without titania particles. Figure $7 \mathrm{~b}$ reveals that conversion of phenol remained constant as a function of time during photolysis, which indicates that a decrease in phenol concentration at $366 \mathrm{~nm}$ in silica-titania composites is due to photocatalytic degradation of phenol rather than due to photolytic degradation. Figure 7c and Figure 7d demonstrate that our photomicroreactor is well suited for photocatalytic degradation of phenol over titania. The concentration of phenol decreased as function of time on the channel walls over titania at a constant flow rate of $12.4 \mu \mathrm{L} / \mathrm{min}$. The phenol concentration remained constant within 10 min and the reactor system henceforth operated at steady state.
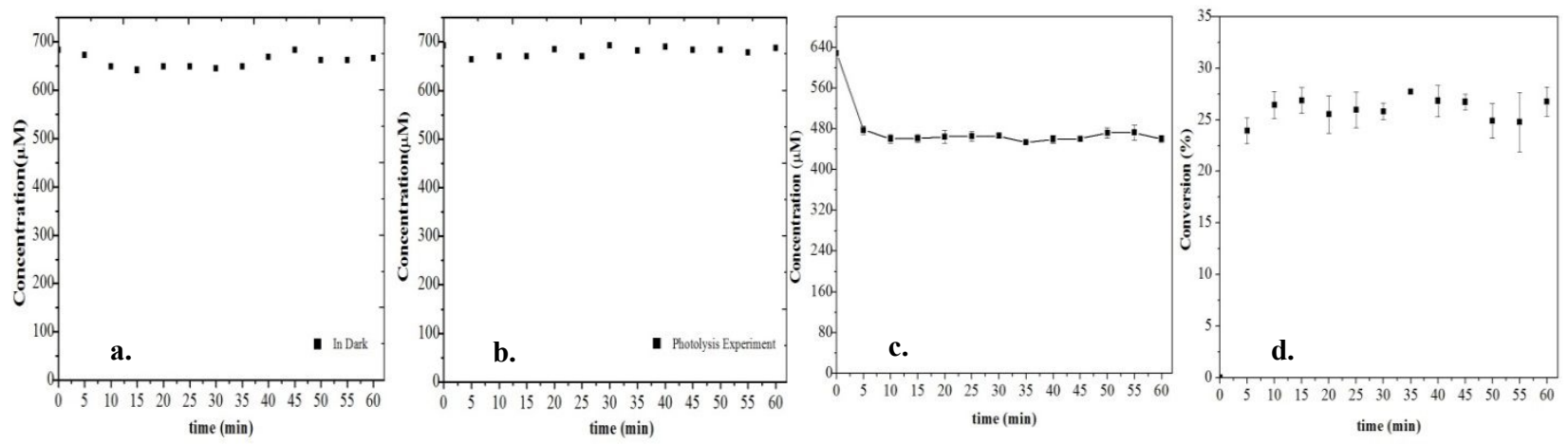

Figure 7. a.Time variation of the concentration of aqueous phenol solution contained in an aerogel channel in the dark. The initial concentration of phenol was $680 \mu \mathrm{M}$. b. Time variation of the concentration of aqueous phenol solution contained in a native silica aerogel channel without photocatalyst under illumination at $366 \mathrm{~nm}$. The initial concentration of phenol was $680 \mu \mathrm{M}$ channel. c. Time variation of the concentration of aqueous phenol solution contained in a silica-titania aerogel channel under illumination at $366 \mathrm{~nm}$. The initial concentration of phenol was $680 \mu \mathrm{M}$ channel. d. Time variation of the percent conversion of phenol over titania. (Channel size: $1.1 \mathrm{~mm} \mathrm{X} 4.1 \mathrm{~cm}, \mathrm{C}_{\mathrm{A}}{ }^{\mathrm{o}}=680 \mu \mathrm{M}, \mathrm{Q}=12.4 \mu \mathrm{L} / \mathrm{min}, \tau=2.95 \mathrm{~min}$ )

\section{CONCLUSIONS}

We have demonstrated a novel type of microphotoreactor with integrated optofluidic waveguide which is formed by a liquid-filled channel fabricated in a monolithic aerogel block. The unique optical properties of aerogels - particularly, 
their low refractive index - allows them to be used as the cladding material of TIR-based optofluidic waveguides with aqueous liquid cores that do not require any additional coatings. Post-synthesis modification of the prepared aerogel monoliths rendered the aerogel surface hydrophobic. Thus, aqueous solutions of methylene blue and phenol serving both as the waveguide core liquid and the reaction medium could be confined inside a channel embedded in the aerogel, without being adsorbed on the channel walls or compromising the monolithic structure of the aerogel. We directly visualized TIR-assisted light propagation along liquid-filled channels fabricated in aerogel blocks and verified that light could be efficiently guided even along paths with a non-linear geometry. Subsequently, we have successfully shown light-driven photolysis of methylene blue by the light guided along the full length of the photoreactor channel. For the studied range of the incident light power, quantitative analysis of the dependence of the relative conversion of the dye on the incident power indicated a linear trend. Lastly, photocatalytic activity and kinetic properties for photocatalytic degradation of a compound (phenol) in the fabricated channels with embedded titania particles using light delivered by waveguiding were investigated by a constant flow rate. It was successfully demonstrated that our photomicroreactor is well suited for photocatalytic degradation of phenol over titania.

\section{REFERENCES}

[1] H. Eskandarloo, and A. Badiei, "Fabrication of an inexpensive and high efficiency microphotoreactor using CO2 laser technique for photocatalytic water treatment applications," Environmental Technology, 36(8), 10631073 (2015).

[2] E. E. Coyle, and M. Oelgemoller, "Micro-photochemistry: photochemistry in microstructured reactors. The new photochemistry of the future?," Photochem Photobiol Sci, 7(11), 1313-22 (2008).

[3] H. Lu, M. A. Schmidt, and K. F. Jensen, "Photochemical reactions and on-line UV detection in microfabricated reactors," Lab Chip, 1(1), 22-8 (2001).

[4] X. He, R. Chen, X. Zhu et al., "Optofluidics-Based Membrane Microreactor for Wastewater Treatment by Photocatalytic Ozonation," Industrial \& Engineering Chemistry Research, 55(31), 8627-8635 (2016).

[5] G. O. S. Williams, J. S. Y. Chen, T. G. Euser et al., "Photonic crystal fibre as an optofluidic reactor for the measurement of photochemical kinetics with sub-picomole sensitivity," Lab on a Chip, 12(18), 3356-3361 (2012).

[6] A. M. Cubillas, X. Jiang, T. G. Euser et al., "Photochemistry in a soft-glass single-ring hollow-core photonic crystal fibre," Analyst, 142(6), 925-929 (2017).

[7] N. Wang, X. Zhang, Y. Wang et al., "Microfluidic reactors for photocatalytic water purification," Lab on a Chip, 14(6), 1074-1082 (2014).

[8] X. Li, H. Wang, K. Inoue et al., "Modified micro-space using self-organized nanoparticles for reduction of methylene blue," Chemical Communications(8), 964-965 (2003).

[9] Y. Matsushita, N. Ohba, S. Kumada et al., "Photocatalytic reactions in microreactors," Chemical Engineering Journal, 135, Supplement 1, S303-S308 (2008).

[10] R. Gorges, S. Meyer, and G. Kreisel, "Photocatalysis in microreactors," Journal of Photochemistry and Photobiology A: Chemistry, 167(2-3), 95-99 (2004).

[11] L. Lei, N. Wang, X. M. Zhang et al., "Optofluidic planar reactors for photocatalytic water treatment using solar energy," Biomicrofluidics, 4(4), 43004 (2010).

[12] S. S. Ahsan, A. Gumus, and D. Erickson, "Redox mediated photocatalytic water-splitting in optofluidic microreactors," Lab Chip, 13(3), 409-14 (2013).

[13] Z. Meng, X. Zhang, and J. Qin, "A high efficiency microfluidic-based photocatalytic microreactor using electrospun nanofibrous TiO2 as a photocatalyst," Nanoscale, 5(11), 4687-4690 (2013).

[14] Y. Özbakır, A. Jonas, A. Kiraz et al., "Aerogels for Optofluidic Waveguides,” Micromachines, 8(4), 98 (2017).

[15] B. Yalizay, Y. Morova, K. Dincer et al., "Versatile liquid-core optofluidic waveguides fabricated in hydrophobic silica aerogels by femtosecond-laser ablation," Optical Materials, 47, 478-483 (2015).

[16] Y. Özbakır, A. Jonáš, A. Kiraz et al., "Total internal reflection-based optofluidic waveguides fabricated in aerogels," Journal of Sol-Gel Science and Technology, 1-13 (2017). 
[17] C. Dette, M. A. Pérez-Osorio, C. S. Kley et al., "TiO2 Anatase with a Bandgap in the Visible Region," Nano Letters, 14(11), 6533-6538 (2014).

[18] G. Zu, J. Shen, W. Wang et al., "Silica-Titania Composite Aerogel Photocatalysts by Chemical Liquid Deposition of Titania onto Nanoporous Silica Scaffolds," ACS Applied Materials \& Interfaces, 7(9), 5400-5409 (2015).

Proc. of SPIE Vol. $10535105352 \mathrm{C}-10$

Downloaded From: https://www.spiedigitallibrary.org/conference-proceedings-of-spie on 18 Jun 2019 Terms of Use: https://www.spiedigitallibrary.org/terms-of-use 